\title{
What happens when laboratory reference ranges change?
}

\author{
Duncan J. Topliss MD
}

Cite as: CMAJ 2020 May 4;192:E481-2. doi: 10.1503/cmaj.200511

See related article at www.cmaj.ca/lookup/doi/10.1503/cmaj.191663

$\mathrm{n}$ related research, Symonds and colleagues ${ }^{1}$ consider the effects of a change in the laboratory reference range for serum thyroid-stimulating hormone (TSH), a common analyte, on clinical practice in Alberta, Canada. Without any change in TSH assay, and thus no systematic change in actual TSH results, the upper limit of the reference range of TSH was changed from 6 $\mathrm{mIU} / \mathrm{L}$ to $4 \mathrm{mIU} / \mathrm{L}$ to improve accuracy and to harmonize the TSH reference ranges across the province. Because there was a single payer for laboratory tests (the governmental health system) and a single laboratory providing the tests in the Calgary region, Symonds and colleagues were able to consider both TSH assay volume and levothyroxine prescription rates over time in the Calgary region and compare these in the period before and after the change in a time series analysis. They found a clear increase in TSH assay volume and a correlated increase in levothyroxine prescriptions consequent to the change in TSH reference range. These findings imply that the change in reference range led more clinicians to consider either that patients had developed biochemical subclinical hypothyroidism (raised TSH with normal range free thyroxine), which led to commencement of levothyroxine treatment, or that patients were failing to respond to an existing levothyroxine dose, which led to increased dosing. This unanticipated clinical effect of a simple change in reference range illustrates the importance of clear communication across the clinical-laboratory interface.

Although clinicians understand the importance of reference ranges for the interpretation of laboratory results and use them in daily practice, few concern themselves with how such ranges are constructed. They may believe that these ranges have been rigorously established and are therefore always robust. Clinical biochemists indeed take great care in providing ranges that are as robust as possible, but there are substantial difficulties in establishing reliable reference ranges. ${ }^{2}$ Construction of reference ranges requires sufficient numbers of healthy individuals distributed over the range of ages and genders for which the reference range is to be provided; a decision as to the range, usually the central $95 \%$ of results (i.e., $2.5 \%$ to $97.5 \%$ ); and an appropriate statistical technique, either parametric or nonparametric, to determine the range. When constructing reference ranges, it's also important to

\section{KEY POINTS}

- Although clinicians understand the importance of reference ranges for the interpretation of laboratory results, they may not consider that a borderline result outside the analytically valid reference range sometimes requires verification or surveillance rather than immediate prescribing action.

- Thyroid-stimulating hormone (TSH) levels exhibit a circadian variation, may vary with age and are affected by iodine intake, medication and smoking.

- Patients with biochemical subclinical hypothyroidism - i.e., a level above the upper bound of the normal reference range ( $4 \mathrm{mIU} / \mathrm{L})-$ may have no symptoms, or nonspecific symptoms that are common in the general population without thyroid dysfunction.

- It may be appropriate to observe TSH levels up to $10 \mathrm{mIU} / \mathrm{L}$ without beginning treatment, in some clinical contexts.

- If a decision is made to change a test's reference range, context and education should be provided by clinical chemists for all clinicians, and advice on interpretation of the changed test could be "boilerplated" to the laboratory report with a hot link to more detailed information available online.

define who is "healthy" and to consider the importance of subclinical disease states. When doing this for thyroid function tests, excluding results from antithyroid antibody-positive individuals is usual, but any workable range must result in some analytically abnormal results not associated with any disease state.

Whether a reference range can be harmonized across several different assay platforms for the same analyte must also be decided. For TSH, assay harmonization may be compromised by variation in the epitopes in the TSH molecule to which antibodies have been raised in different TSH assays, ${ }^{3}$ resulting in different analyte signal generation in different assays for the same serum. Furthermore, a TSH reference range has its own specific challenges: TSH exhibits a circadian variation; the level of the hormone varies with age; ${ }^{4}$ and the level is influenced by iodine intake, medication, smoking and occult thyroid autoimmune disease. ${ }^{5}$ Finally, TSH immunoreactivity can be discordant to bioreactivity because of variation in glycosylation of the TSH molecule, which in turn affects TSH receptor binding and action. 
Clinical biochemists may not realize that busy clinicians especially those in primary care who order a wide variety of tests - may lack knowledge of the limitations of reference ranges and might not appreciate the significance of a reference range change, if they notice it at all. The clinical biochemist may consider their job is done when an analytically valid reference range has been provided. The clinician may consider that they can take any result outside the range as clinically important. They may not consider that a borderline result outside the analytically valid reference range sometimes requires verification or surveillance rather than immediate prescribing action.

Patients with biochemical subclinical hypothyroidism may have no symptoms; if symptoms are present, they may be nonspecific symptoms that are common in the general population without thyroid dysfunction. Screening or case-finding of asymptomatic thyroid dysfunction is not recommended in primary care. ${ }^{6}$ Treatment of subclinical thyroid dysfunction may have no clinical benefit in the short term. ${ }^{7}$ Indeed, treatment may have adverse effects and be associated with cardiac and skeletal risks. ${ }^{8}$ Moreover, in older adults, application of a single reference range that is not age specific may lead to inappropriate overtreatment.

A 2017 study ${ }^{9}$ showed that beginning reflex testing of free $T_{4}$ using a TSH cut-off of 1-2 mIU/L greater than the reference range had only a minimal effect on case-finding of clinically important hypothyroidism, suggesting that TSH values of up to $6.0 \mathrm{mIU} / \mathrm{L}$ may not require clinical action. Some authors concluded that, with careful consideration of clinical context, it may be appropriate to observe TSH levels up to $10 \mathrm{mIU} / \mathrm{L}$ without beginning treatment. ${ }^{10}$ Although epidemiological data from 2010 suggested long-term adverse cardiovascular consequences of subclinical hypothyroidism, ${ }^{11}$ many experts consider that older people may tolerate apparent subclinical hypothyroidism well. ${ }^{12}$ Individual patient context, age and the degree of elevation of TSH should be considered in the decision to start levothyroxine therapy in subclinical hypothyroidism. ${ }^{10}$

Continuing and proactive dialogue between clinical biochemists and expert clinicians is needed to mitigate potential clinical consequences of any intended assay or reference range change. If a decision is made to change these, context and education should be provided for all clinicians. This dialogue might be conducted efficiently by the clinical biochemist contacting, when necessary, a limited number of nominated key clinicians in the relevant areas of expertise, who should reflect on any clinical implications, seek the opinion of colleagues as needed and then provide prompt feedback. Agreed advice on interpretation of the changed test could be "boilerplated" to the laboratory report and linked to more detailed information available online. Symonds and colleagues, by showing clearly the unanticipated overtreatment effect of a change in the laboratory reference range for $\mathrm{TSH}$, have shown the continuing importance of communication between biochemists and front-line clinicians.

\section{References}

1. Symonds C, Kline G, Gjata I, et al. Levothyroxine prescribing and laboratory test use after a minor change in reference range for thyroid-stimulating hormone. CMAJ 2020;192:E469-75.

2. Katayev A, Balciza C, Seccombe DW. Establishing reference intervals for clinical laboratory test results: Is there a better way? Am J Clin Pathol 2010;133:180-6.

3. Lee GR, Griffin A, Halton K, et al. Generating method-specific Reference Ranges - A harmonious outcome? Pract Lab Med 2017;9:1-11.

4. Raverot V, Bonjour M, Payrat JA, et al. Age- and sex-specific TSH upper-limit reference intervals in the general French population: there is a need to adjust our actual practices. J Clin Med 2020;9:E792. doi: 10.3390/jcm9030792.

5. Razvi S, Hostalek U. Therapeutic challenges in the application of serum thyroid stimulating hormone testing in the management of patients with hypothyroidism on replacement thyroid hormone therapy: a review. Curr Med Res Opin 2019;35: 1215-20.

6. Birtwhistle R, Morissette K, Dickinson JA, et al.; Canadian Task Force on Preventive Health Care. Recommendation on screening adults for asymptomatic thyroid dysfunction in primary care. CMAJ 2019;191:E1274-80.

7. Stott DJ, Rodondi N, Kearney PM, et al.; TRUST Study Group. Thyroid hormone therapy for older adults with subclinical hypothyroidism. N Engl J Med 2017;376: 2534-44.

8. Taylor PN, Iqbal A, Minassian C, et al. Falling threshold for treatment of borderline elevated thyrotropin levels - balancing benefits and risks: evidence from a large community-based study. JAMA Intern Med 2014;174:32-9.

9. Henze M, Brown SJ, Hadlow NC, et al. Rationalizing thyroid function testing: Which TSH cutoffs are optimal for testing free T4? J Clin Endocrinol Metab 2017;102:4235-41.

10. Sawka AM, Cappola AR, Peeters RP, et al. Patient context and thyrotropin levels are important when considering treatment of subclinical hypothyroidism. Thyroid 2019;29:1359-63.

11. Rodondi N, den Elzen WPJ, Bauer DC, et al.; Thyroid Studies Collaboration. Subclinical hypothyroidism and the risk of coronary heart disease and mortality. JAMA 2010;304:1365-74.

12. Pasqualetti G, Tognini S, Polini A, et al. Is subclinical hypothyroidism a cardiovascular risk factor in the elderly? J Clin Endocrinol Metab 2013;98:2256-66.

Competing interests: In the last 36 months, Duncan Topliss has served as a speaker for Boehringer Ingelheim, Eisai, Genzyme, Novartis and Novo Nordisk, and as an advisory board member for Eisai. He has received research funding from Bayer, Eisai and Exelixis.

This article was solicited and has not been peer reviewed.

Affiliation: Department of Endocrinology and Diabetes, The Alfred; and Monash University, Melbourne, Australia

Correspondence to: Duncan Topliss, duncan.topliss@monash.edu 